\title{
A Construction for Constant-Composition Codes
}

\author{
Yang Ding
}

\begin{abstract}
By employing the residue polynomials, we give a construction of constant-composition codes. This construction generalizes the one proposed by Xing [16]. It turns out that when $d=3$ this construction gives a lower bound of constantcomposition codes improving the one in [10] for some case. Moreover, for $d>3$, we give a lower bound on maximal size of constant-composition codes. In particular, our bound for $d=5$ gives the best possible size of constant-composition codes up to magnitude.
\end{abstract}

Index Terms - constant-composition codes, genus, residue polynomial, rational function fields.

\section{INTRODUCTION}

Constant-composition codes are a subclass of constant weight codes, in which both weight restrict and element composition restrict are involved. The class of constantcomposition codes have attracted recent interest due to its numerous applications, such as in determining the zero error decision feedback capacity of discrete memoryless channels [15], multiple-access communications [8], spherical codes for modulation [9], DNA codes [11], powerline communications [2], and frequency hopping [3].

One of the most fundamental problem in coding theory is the problem of determining the maximum size of a block code, given its length and minimum distance. The problem of determining the maximum size of a constant-composition code is much less understood than the constant-weight and linear cases. In the recent years, researches consider the problems of maximizing the size of a constant-composition code (see [1], [10], [13]), and constructing optimal codes to achieve these bounds (see [4], [5], [6], [7], [14]). In this paper, we give a construction for constant-composition codes then produce a lower bound on constant-composition codes for arbitrary given minimum distance. We show that when $q=3$ and $d=5$, our bound gives the best possible size of constant-composition codes up to magnitude. As far as we know, except for the bound given in this paper, there is no bounds on $d>3$ so far.

This correspondence is organized as follows. In Section II, we introduce some basic definitions and notations. We also review some basic properties which will be used in this correspondence. The main construction is presented in Section III. In Section IV, Theorem 1 in section II are used to obtain some good lower bounds on constant-composition codes.

This work was supported by the China Scholarship Council.

The author is with the Department of Mathematics, Southeast University, Nanjing, 210096, People's Republic of China (e-mail: PG23067461@ntu.edu.sg). This work was carried out while the author was studying in Division of Mathematical Sciences, School of Physical and Mathematical Sciences, Nanyang Technological University, Singapore under the exchange program.

\section{PRELIMINARY}

We use the standard notations for codes as follows. Let $\mathrm{Z}_{q}$ denote the set $\{0,1, \cdots, q-1\}$, and let $\mathrm{Z}_{q}^{n}$ be the set of all $n$-tuples over $\mathrm{Z}_{q}$, where $q$ is a positive integer. Let $V_{n,\left[\omega_{0}, \omega_{1}, \cdots, \omega_{q-1}\right]}(q)$ denote the set of $n$-tuples over $\mathrm{Z}_{q}$ of the fixed composition $\left[\omega_{0}, \omega_{1}, \cdots, \omega_{q-1}\right]$, i.e., the number of 0 's, 1 's, $\cdots, q-1$ 's in the $n$ tuple over $\mathrm{Z}_{q}$ is given by $\omega_{0}, \omega_{1}, \cdots, \omega_{q-1}$, respectively, where $n=\omega_{0}+\omega_{1}+\cdots+\omega_{q-1}$. It is obvious that $V_{n,\left[\omega_{0}, \omega_{1}, \cdots, \omega_{q-1}\right]}(q)$ contains $\left(\begin{array}{c}n \\ \omega_{0}, \omega_{1}, \cdots, \omega_{q-1}\end{array}\right)$ elements. An $\left(n, M, d,\left[\omega_{0}, \quad \omega_{1}, \cdots, \quad \omega_{q-1}\right]\right)_{q}$ constantcomposition code $C$ is a subset of $V_{n,\left[\omega_{0}, \omega_{1}, \cdots, \omega_{q-1}\right]}(q)$ with size $M$ and minimum Hamming distance $d$. We use $A_{q}\left(n, d,\left[\omega_{0}, \omega_{1}, \cdots, \omega_{q-1}\right]\right)$ to denote the maximum size of an $\left(n, M, d,\left[\omega_{0}, \omega_{1}, \cdots, \omega_{q-1}\right]\right)_{q}$ constant-composition code.

In order to establish our results in this correspondence, we need the following Lemmas.

Let $\operatorname{gcd}(\alpha, \beta)$ be the greatest common divisor of the positive integers $\alpha$ and $\beta$. Denote

$$
Q=\prod_{\substack{p \text { is prime } \\ p \leq q-1}} p, \text { for } q \geq 3
$$

and

$L(s, q)=\min \{l: l \geq s$ and $\operatorname{gcd}(l, Q)=1\}$, for $0 \leq s \leq Q-1\}$.

Lemma 1: (cf. [10])

$A_{q}\left(n, 3,\left[\omega_{0}, \cdots, \omega_{q-1}\right]\right) \geq\left(\begin{array}{c}n \\ \omega_{0}, \cdots, \omega_{q-1}\end{array}\right) /\left(n+\Gamma\left(t_{n}, q\right)\right)$,

where $t_{n}$ is the least nonnegative integer such that $t_{n} \equiv n($ $\bmod Q)$, and $\Gamma\left(t_{n}, q\right)=L\left(t_{n}, q\right)-t_{n}$.

Lemma 2: (cf. [10]) Let $Q$ be given by (1). If $\operatorname{gcd}(n, Q)=$ 1 , then

$$
A_{q}\left(n, 3,\left[\omega_{0}, \cdots, \omega_{q-1}\right]\right) \geq\left(\begin{array}{c}
n \\
\omega_{0}, \cdots, \omega_{q-1}
\end{array}\right) / n
$$

Lemma 3: (cf. [10]) For $q=3$,

$A_{3}\left(n, 3,\left[\omega_{0}, \omega_{1}, \omega_{2}\right]\right)$

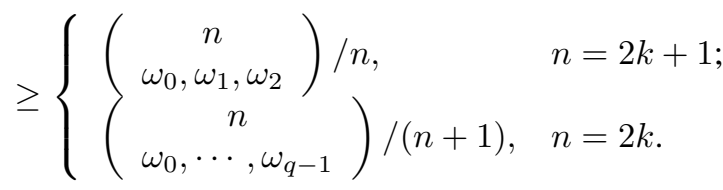


In this correspondence, bound (5) is improved for even length.

For a constant-composition code with length $n$, minimum distance at least $d$, and constant composition $\left[\omega_{0}, \cdots, \omega_{q-1}\right]$, denote $\delta=\lfloor(d-1) / 2\rfloor$. Then $\delta<\omega_{1}+\cdots+\omega_{q-1}$.

Lemma 4: (cf. [10]) For any fixed $i$ where $0 \leq i \leq q-1$, we have

$$
\begin{aligned}
& A_{q}\left(n, d,\left[\omega_{0}, \cdots, \omega_{q-1}\right]\right) \\
& \quad \leq\left(\begin{array}{c}
n \\
\omega_{0}, \cdots, \omega_{q-1}
\end{array}\right) /\left(\begin{array}{c}
\omega_{i}+\delta \\
\omega_{i}, \delta_{i, 0}, \cdots, \delta_{i, q-1}
\end{array}\right)
\end{aligned}
$$

where $\delta_{i, j}, 1 \leq j \leq q-1$ are nonnegative integers such that $\delta_{i, i}=0, \delta_{i, 0}+\cdots+\delta_{i, q-1}=\delta$, and $\delta_{i, l} \leq \omega_{l}$ for $0 \leq l \leq q-1$.

In this paper, we show that when $d=5$, we give a lower bound have the same magnitude with bound (6).

\section{CONSTRUCTION OF CODES}

In this section, we generalize the construction that is proposed by Xing [16]. Let $r$ be a prime power. We denote by $\mathbf{F}_{r}$ the finite field with $r$ elements. We label all elements of $\mathbf{F}_{r}$

$$
\mathbf{F}_{r}=\left\{\alpha_{0}=0, \alpha_{1}, \cdots, \alpha_{r-1}\right\} .
$$

For a positive integer $m$, consider the residue ring of polynomials

$$
\mathbf{F}_{r}[x] /\left(x^{m}\right) .
$$

It is a finite ring and has $r^{m}$ elements. All invertible elements of this ring form a multiplicative group, denoted by $\left(\mathbf{F}_{r}[x] /\left(x^{m}\right)\right)^{*}$. It is a finite abelian group. The quotient group

$$
\left(\mathbf{F}_{r}[x] /\left(x^{m}\right)\right)^{*} / \mathbf{F}_{r}^{*}
$$

is a finite abelian group with $r^{m-1}$ elements.

Let $e$ is a positive integer, for a prime $p$, we define

$$
\mu_{p}(e)=\left\{\begin{array}{cc}
e & \text { if } p \mid e \\
e-1 & \text { otherwise. }
\end{array}\right.
$$

Theorem 1: Let $q \geq 3$ be a integer and let $r$ be a power of $p$ for a prime $p$. If $p \geq q$, then for any positive integer $d_{0}$ satisfying $1 \leq d_{0} \leq r-2$, there exist a $q$-ary $\left(r, M, \geq \mu_{p}\left(d_{0}\right)+2,\left[\omega_{0}, \quad \omega_{1}, \cdots, \omega_{q-1}\right]\right)$ constantcomposition code with

$$
M \geq\left(\begin{array}{c}
r \\
\omega_{0}, \cdots, \omega_{q-1}
\end{array}\right) / r^{d_{0}-1}
$$

Proof. Consider the map

$$
\begin{gathered}
\pi: V_{r,\left[\omega_{0}, \omega_{1}, \cdots, \omega_{q-1}\right]}(q) \rightarrow\left(\mathbf{F}_{r}[x] / x^{d_{0}}\right)^{*} / \mathbf{F}_{r}^{*} \\
\left(c_{1}, c_{2}, \cdots, c_{r}\right) \mapsto \prod_{i=1}^{r-1}\left(x-\alpha_{i}\right)^{c_{i}} .
\end{gathered}
$$

By the Pigeonhole Principle, it is clear that we can find one element $\overline{f(x)}$ from this quotient group such that it has at least

$$
\begin{gathered}
\left(\begin{array}{c}
r \\
\omega_{0}, \cdots, \omega_{q-1} \\
r \\
\omega_{0}, \cdots, \omega_{q-1}
\end{array}\right) / r^{d_{0}-1} \text { pre-images, i.e., } \#\left(\pi^{-1}(\overline{f(x)})\right) \geq \\
C=\pi^{-1}(\overline{f(x)}) .
\end{gathered}
$$

We are going to show that $C$ is a code with the desired parameters. The length of $C$ is clearly $r$. The remaining thing is to show that the minimum distance is at least $\mu_{p}\left(d_{0}\right)+2$.

Let $\mathbf{u}=\left(u_{1}, u_{2}, \cdots, u_{r}\right)$ and $\mathbf{v}=\left(v_{1}, v_{2}, \cdots, v_{r}\right)$ be two distinct codewords of $C$. Then, $\pi(\mathbf{u})=\pi(\mathbf{v})=\overline{f(x)}$. This implies that in the group $\left(\mathbf{F}_{r}[x] /\left(x^{d_{0}}\right)\right)^{*}$, the element

$$
\frac{\prod_{i=1}^{r-1}\left(x-\alpha_{i}\right)^{u_{i}}}{\prod_{i=1}^{r-1}\left(x-\alpha_{i}\right)^{v_{i}}}
$$

is equal to $\alpha$ for some nonzero element $\alpha$ of $\mathbf{F}_{r}^{*}$.

Put

$$
z:=\frac{\prod_{i=1}^{r-1}\left(x-\alpha_{i}\right)^{u_{i}}}{\prod_{i=1}^{r-1}\left(x-\alpha_{i}\right)^{v_{i}}} \in \mathbf{F}_{r}(x)
$$

It is clear that $\mathrm{z}$ is not a constant as $\mathbf{u} \neq \mathbf{v}$.Then the principal divisor of $z$ is equal to

$$
\operatorname{div}(z)=\sum_{i=1}^{r-1}\left(u_{i}-v_{i}\right) P_{i}+\left(\sum_{i=1}^{r-1}\left(v_{i}-u_{i}\right)\right) P_{\infty}
$$

where $P_{i}$ is the place corresponding to $\left(x-\alpha_{i}\right)$ for all $1 \leq$ $i \leq r-1$, and $P_{\infty}$ is corresponding to the infinite place.

Consider the field extension $\mathbf{F}_{r}(x) / \mathbf{F}_{r}(z)$ of degree

$$
\left(\sum_{i=1}^{r-1}\left|u_{i}-v_{i}\right|+\left|\sum_{i=1}^{r-1}\left(v_{i}-u_{i}\right)\right|\right) / 2
$$

where $|$.$| stands for the absolute value of a real number. We$ know this extension is separable as $p \geq q$ (cf.[16]).

For $1 \leq i \leq r-1$, whenever $u_{i}-v_{i} \neq 0$, the place $P_{i}$ has the ramification index $\left|u_{i}-v_{i}\right|$ in the extension $\mathbf{F}_{r}(x) / \mathbf{F}_{r}(z)$ and hence the different exponent $D_{P_{i}}$ of $P_{i}$ is at least $\left|u_{i}-v_{i}\right|-1$ (see [12]).

The fact that $z$ is equal to $\alpha$ in the $\operatorname{group}\left(\mathbf{F}_{r}[x] /\left(x^{d_{0}}\right)\right)^{*}$ implies that $P_{0}$ is a zero of $z-\alpha$ with multiplicity at least $d_{0}$. Hence, the ramification index of the place $P_{0}$ with respect to the extension $\mathbf{F}_{r}(x) / \mathbf{F}_{r}(z)$ is at least $d_{0}$, therefore, the different exponent $D_{P_{0}} \geq d_{0}-1$. In particular, if $p \mid d_{0}$, by Dedekind's Different Theorem, we obtain $D_{P_{0}} \geq d_{0}$. So, $D_{P_{0}} \geq \mu_{p}\left(d_{0}\right)$.

Let

$$
S=\left\{i \in\{1,2, \cdots, r-1\}: u_{i} \neq v_{i}\right\}
$$

and let $\omega$ be the distance between $\mathbf{u}$ and $\mathbf{v}$.

1 If $u_{r}=v_{r}$, then $|S|=\omega$ and $\sum_{i=1}^{r-1}\left(v_{i}-u_{i}\right)=0$. Hence

$$
\sum_{i \in S} D_{P_{i}} \geq \sum_{i \in S}\left(\left|u_{i}-v_{i}\right|-1\right)=\left(\sum_{i=1}^{r-1}\left|u_{i}-v_{i}\right|\right)-\omega
$$

By (7) the different exponent of $P_{\infty}$ with respect to the extension $\mathbf{F}_{r}(x) / \mathbf{F}_{r}(z)$ at least 0 . The genera $g\left(\mathbf{F}_{r}(x)\right)$ 
and $g\left(\mathbf{F}_{r}(z)\right)$ are both equal to 0 . Thus, by the Huiwitz genus formula (see [12]), we have

$$
\begin{aligned}
-2 & =2 g\left(\mathbf{F}_{r}(x)\right)-2 \\
& =\left(2 g\left(\mathbf{F}_{r}(z)\right)-2\right)\left[\mathbf{F}_{r}(x): \mathbf{F}_{r}(z)\right]+\sum_{P} D_{P} \\
& \geq-2\left[\mathbf{F}_{r}(x): \mathbf{F}_{r}(z)\right]+\sum_{i \in S} D_{P_{i}}+D_{P_{0}}+D_{P_{\infty}} \\
\geq & -2\left(\left(\sum_{i=1}^{r-1}\left|u_{i}-v_{i}\right|\right) / 2\right)+\left(\sum_{i=1}^{r-1}\left|u_{i}-v_{i}\right|\right) \\
& -\omega+\mu_{p}\left(d_{0}\right) \\
= & \mu_{p}\left(d_{0}\right)-\omega .
\end{aligned}
$$

So, $\omega \geq \mu_{p}\left(d_{0}\right)+2$.

2 If $u_{i} \neq v_{i}$, then $|S|=\omega-1$. Hence

$$
\sum_{i \in S} D_{P_{i}} \geq \sum_{i \in S}\left(\left|u_{i}-v_{i}\right|-1\right)=\left(\sum_{i=1}^{r-1}\left|u_{i}-v_{i}\right|\right)-\omega+1 .
$$

By (7) the different exponent of $P_{\infty}$ with respect to the extension $\mathbf{F}_{r}(x) / \mathbf{F}_{r}(z)$ at least $\left|\sum_{i=1}^{r-1}\left(v_{i}-u_{i}\right)\right|-1$. Thus by the Huiwitz genus formula, we have

$$
\begin{aligned}
-2 & =2 g\left(\mathbf{F}_{r}(x)\right)-2 \\
& =\left(2 g\left(\mathbf{F}_{r}(z)\right)-2\right)\left[\mathbf{F}_{r}(x): \mathbf{F}_{r}(z)\right]+\sum_{P} D_{P} \\
\geq & -2\left[\mathbf{F}_{r}(x): \mathbf{F}_{r}(z)\right]+\sum_{i \in S} D_{P_{i}}+D_{P_{0}}+D_{P_{\infty}} \\
\geq & -2\left(\left(\sum_{i=1}^{r-1}\left|u_{i}-v_{i}\right|+\left|\sum_{i=1}^{r-1}\left(v_{i}-u_{i}\right)\right|\right) / 2\right)+ \\
& \left(\sum_{i=1}^{r-1}\left|u_{i}-v_{i}\right|\right)-\omega+\mu_{p}\left(d_{0}\right)+\left|\sum_{i=1}^{r-1}\left(v_{i}-u_{i}\right)\right|-1 \\
= & \mu_{p}\left(d_{0}\right)-\omega .
\end{aligned}
$$

So, $\omega \geq \mu_{p}\left(d_{0}\right)+2$.

The desired result follows.

\section{SOME EXAMPLES FOR LOWER BOUND ON CONSTANT-COMPOSITION CODES}

Now, we can get some improved lower bounds for constantcomposition codes from Theorem 1. We adopt the notations and terminologies in the previous section and consider the quotient group

$$
\left(\mathbf{F}_{r}[x] / x^{d_{0}}\right)^{*} / \mathbf{F}_{r}^{*}
$$

Example 1. Consider $d_{0}=2$

(1) For the case $p \geq q \geq 3, \mu_{p}\left(d_{0}\right)=1$, the group $\left(\mathbf{F}_{r}[x] /\left(x^{2}\right)\right)^{*} /\left(\mathbf{F}_{r}\right)^{*}$ has $r$ elements. By Theorem 1 we can get a constant-composition code with parameters $\left(r, M, d,\left[\omega_{0}, \omega_{1}, \cdots, \omega_{q-1}\right]\right)$, where $d \geq 3$, and

$$
M \geq\left(\begin{array}{c}
r \\
\omega_{0}, \cdots, \omega_{q-1}
\end{array}\right) / r .
$$

Then we obtain

$$
A_{q}\left(r, 3,\left[\omega_{0}, \cdots, \omega_{q-1}\right]\right) \geq\left(\begin{array}{c}
r \\
\omega_{0}, \cdots, \omega_{q-1}
\end{array}\right) / r .
$$

The bound in this case achieves the one given in Lemma 2 for codes with odd length.

(2) Now we consider the code of even length. Let $q=3$, $2 \mid r$, from the first part proof of theorem 1, we know that we can get a constant-composition code of size $\geq\left(\begin{array}{c}r \\ \omega_{0}, \omega_{1}, \omega_{2}\end{array}\right) / r$, then we want to show this code has minimum distance $\geq 3$. For two distinct codewords $\mathbf{u}=\left(u_{1}, u_{2}, \cdots, u_{r}\right)$ and $\mathbf{v}=\left(v_{1}, v_{2}, \cdots, v_{r}\right)$, similar to Theorem 1, consider

$$
\frac{u(x)}{v(x)}:=\frac{\prod_{i=1}^{r-1}\left(x-\alpha_{i}\right)^{u_{i}}}{\prod_{i=1}^{r-1}\left(x-\alpha_{i}\right)^{v_{i}}} \equiv \alpha \bmod \left(x^{2}\right) .
$$

for some nonzero element $\alpha$ of $\mathbf{F}_{r}^{*}$.

1 If $u_{r} \neq v_{r}$, the distance between $\mathbf{u}$ and $\mathbf{v}$ is 2 if and only if $\frac{u(x)}{v(x)}=\left(x-\alpha_{i}\right)$ or $\frac{u(x)}{v(x)}=\frac{1}{x-\alpha_{i}}$ for some $i, 1 \leq i \leq r-1$. Both of these two cases are not satisfy (9), so we get $d \geq 3$.

2 If $u_{r}=v_{r}$, it is easy to know that the distance between $\mathbf{u}=\left(u_{1}, u_{2}, \cdots, u_{r}\right)$ and $\mathbf{v}=\left(v_{1}, v_{2}, \cdots, v_{r}\right)$ is 2 if and only if $\frac{u(x)}{v(x)}=\frac{x-\alpha_{i}}{x-\alpha_{j}}$ or $\frac{u(x)}{v(x)}=\frac{\left(x-\alpha_{i}\right)^{2}}{\left(x-\alpha_{j}\right)^{2}}$, for some $i, j, 1 \leq i \neq j \leq r-1$. Since $\operatorname{char} \mathbf{F}_{r}=2$, both of these two cases are not satisfy (9), so we get $d \geq 3$.

Then

$$
A_{3}\left(r, 3,\left[\omega_{0}, \omega_{1}, \omega_{2}\right]\right) \geq\left(\begin{array}{c}
r \\
\omega_{0}, \omega_{1}, \omega_{2}
\end{array}\right) / r
$$

Bound (10) improves the one given in Lemma 3 when the length of code is even.

Example 2. Consider $d_{0}=3$ :

(1) For the case $p=\operatorname{char}\left(\mathbf{F}_{r}\right)=q=3$. Then $p \mid d_{0}$, since $\mu_{p}\left(d_{0}\right)=3$ we get $d \geq 5$. By Theorem 1 , we get a 3 -ary $\left(r, M, 5,\left[\omega_{0}, \omega_{1}, \omega_{2}\right]\right)$ constant-composition code, where

$$
M \geq\left(\begin{array}{c}
r \\
\omega_{0}, \omega_{1}, \omega_{2}
\end{array}\right) / r^{2} .
$$

Hence,

$$
A_{3}\left(r, 5,\left[\omega_{0}, \omega_{1}, \omega_{2}\right]\right) \geq\left(\begin{array}{c}
r \\
\omega_{0}, \omega_{1}, \omega_{2}
\end{array}\right) / r^{2} .
$$

Lemma 3 given a upper bound of constant-composition codes. Now we take $d=5$, then $\delta=\left\lfloor\frac{d-1}{2}\right\rfloor=2$, it is easy to know that there exist $\omega_{i} \geq\lfloor r / q\rfloor$ for $0 \leq i \leq q-1$. So we have

$$
A_{3}\left(r, 5,\left[\omega_{0}, \omega_{1}, \omega_{2}\right]\right) \leq\left(\begin{array}{c}
n \\
\omega_{0}, \omega_{1}, \omega_{2}
\end{array}\right) /\left(\begin{array}{c}
\omega_{i}+2 \\
\omega_{i}, \delta_{i, 0}, \delta_{i, 1}, \delta_{i, 2}
\end{array}\right)
$$

where $\delta_{i, j}$ are nonnegative $\delta_{i, 0}+\delta_{i, 1}+\delta_{i, 2}=2$, we choose $\delta_{i, 0}, \delta_{i, 1}, \delta_{i, 2}$ such that $\left(\begin{array}{c}2 \\ \delta_{i, 0}, \delta_{i, 1}, \delta_{i, 2}\end{array}\right)=2$, then $t(r)=\left(\begin{array}{c}\omega_{i}+2 \\ \omega_{i}, \delta_{i, 0}, \delta_{i, 1}, \delta_{i, 2}\end{array}\right)=\left(\omega_{i}+2\right)\left(\omega_{i}+1\right) \geq$ $\left(\frac{r}{q}+1\right) \frac{r}{q}=O\left(r^{2}\right)$ when $r \rightarrow \infty$, then we obtain an upper bound for constant composition code over $\mathbf{F}_{3}$ of 
minimum distance 5

$$
A_{3}\left(r, 5,\left[\omega_{0}, \omega_{1}, \omega_{2}\right]\right) \leq\left(\begin{array}{c}
r \\
\omega_{0}, \omega_{1}, \omega_{2}
\end{array}\right) / t(r)
$$

where $t(r)=O\left(r^{2}\right)$, compare this upper bound with our lower bound in (9), our lower bound given the best possible size up to magnitude.

(2) For the case $p \geq q \geq 3$ and $p>3$, then we obtain $d \geq 4$ since $\mu_{p}\left(d_{0}\right)=2$, By Theorem 1 , we obtain a $q$-ary $\left(r, M, 4,\left[\omega_{0}, \omega_{1}, \cdots, \omega_{q-1}\right]\right)$ constant-composition code, where

$$
M \geq\left(\begin{array}{c}
r \\
\omega_{0}, \cdots, \omega_{q-1}
\end{array}\right) / r^{2},
$$

and the lower bound

$$
A_{q}\left(r, 4,\left[\omega_{0}, \cdots, \omega_{q-1}\right]\right) \geq\left(\begin{array}{c}
r \\
\omega_{0}, \cdots, \omega_{q-1}
\end{array}\right) / r^{2}
$$

Example 3. Let $d_{0}=5$

(1) For the case $p=q=5$, we get $d \geq 7$, then by Theorem 1 ,

$$
A_{q}\left(r, 7,\left[\omega_{0}, \cdots, \omega_{q-1}\right]\right) \geq\left(\begin{array}{c}
r \\
\omega_{0}, \cdots, \omega_{q-1}
\end{array}\right) / r^{4} .
$$

(2) If $p=>5$ and $3 \leq q \leq p$, we get $d \geq 6$ and lower bound

$$
A_{q}\left(r, 6,\left[\omega_{0}, \cdots, \omega_{q-1}\right]\right) \geq\left(\begin{array}{c}
r \\
\omega_{0}, \cdots, \omega_{q-1}
\end{array}\right) / r^{4} .
$$

\section{Remark 2:}

1 The construction in this paper produces a lower bound on constant-composition codes for arbitrary given minimum distance.

2 As far as we know, except for the bound given in this paper, there are no bounds on $A_{q}\left(n, d,\left[\omega_{0}, \cdots, \omega_{q-1}\right]\right)$, where $d \geq 4$, so far.

\section{ACKNOWLEDGMENT}

The author is grateful to Profs. Keqin Feng, Jianlong Chen and Chaoping Xing for their guidance.

\section{REFERENCES}

[1] Y. M. Chee, A. C. H. Ling, S. Ling and H. Shen, "PBD-Closure of Constant Composition Codes," IEEE Transactions on Information Theory, vol. 53, pp 2685-2692, Aug. 2007.

[2] W.Chu, C.J.Colbourn, and P.Dukes, "Constructions for permutation codes in powerline communnications," Des., Codes, Cryptogr., vol.32, no.1-3, pp.51-64, 2004.

[3] W.Chu, C.J.Colbourn, and P.Dukes, "On constant composition codes," Discr. Appl. Math., vol.154, no.6, pp. 912-926, 2006.

[4] C. S. Ding and J. X. Yin, "Combinatorial constructions of optimal constant-composition codes," IEEE Trans. Inform. Theory, vol. 51, pp. 3671-3674, Oct. 2005.

[5] C. S. Ding and J. X. Yin, "Algebraic constructions of constantcomposition codes," IEEE Trans. Inform. Theory, vol. 51, pp. 15851589, Oct. 2005.

[6] C. S. Ding and J. X. Yin,"A construction of optimal constant composition codes," Designs Codes and Cryptography, vol.40(2), pp. 157-165, Aug. 2006.

[7] C. S. Ding, J. Yuan, "A Family of Optimal Constant-Composition Codes," IEEE Trans. Inform. Theory, vol.51, pp 3668-3671, Oct. 2005.
[8] A.G.D’yachkov, "Random constant composition codes for multiple access channels," Probl. COntr. Inf. Theory/Probl. Upravlen. Teor.Inform. vol.13, no.6, pp.357-369, 1984.

[9] T.Ericson and V.Zinoviev, "Spherical codes generated by binary partitions of symmetric pointsets," IEEE Trans. Inform. Theory, vol.41, pp 107-129, Jan. 1995.

[10] Y. Luo, F. W. Fu, A. J. H. Vinck and W. D. Chen, "On constantcomposition codes over $Z_{q}$," IEEE Trans. Inform. Theory, vol.49, pp.3010-3016, Nov. 2003.

[11] O.Milenkovic and N.Kashyap, On the Design of Codes for DNA (Lecture Notes in Computer Science), Berline, Germany: Springer-Verlag, 2006, vol.3969, pp 100-119.

[12] H. Stichtenoth, Algebraic function fields and codes, Berlin,Germany: Springer-Verlag, 1993.

[13] M. Svanström, P. R. J. Östergôrd and G. T. Bogdanova, "Bounds and Constructions for Ternary Constant-Composition Codes," IEEE Trans. on Inform. Theory, vol. 48, pp. 101-111, Jan. 2002.

[14] M.Svanström, "Constructions of ternary constant-composition codes with weight three," IEEE Trans. Inform. Theory, vol.46, pp.2644-2647, Nov.2000.

[15] I.E.Telatar and R.G.Gallager, "Zero error decision feedback capacity of discrete memoryless channels," in BILCON'90: Proc. 1990 Bilkent Int. Conf. New Trends in Communications, Control and Signal Processing, E.Arikan, Ed. Amsterdam, The Netherlands: Elsevier, 1990, pp. 228 233.

[16] C. P. Xing, "Constructions of codes from residue rings of polynomials," IEEE Trans. Inform. Theory, vol.48, pp.2995-2997, Nov. 2002 